\title{
Surgical Management of Intestinal Failure; Initial Experiences from an Intestinal Failure, Rehabilitation and Transplant Unit in Iran
}

\author{
Hamed Nikoupour, MD¹; Peyman Arasteh, MD¹; Alireza Shamsaeefar, MD'; Mojtaba Shafiekhani, PharmD ${ }^{1,2}$; Ali Mohammad

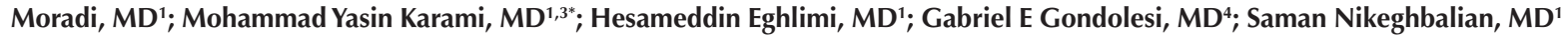 \\ 'Shiraz Transplant Research Center, Shiraz University of Medical Sciences, Shiraz, Iran \\ ${ }^{2}$ Department of Clinical Pharmacy, School of Pharmacy, Shiraz University of Medical Sciences, Shiraz, Iran \\ ${ }^{3}$ Breast Diseases Research Center, Shiraz University of Medical Sciences, Shiraz, Iran \\ ${ }^{4}$ Unidad de Insuficiencia, Rehabilitacion y Trasplante Intestinal, Hospital Universitario Fundación Favaloro, Buenos Aires, Argentina
}

\begin{abstract}
Background: Intestinal failure (IF) is a life-threatening medical condition. The management of IF in low- and middle-income countries without home parenteral nutrition (HPN) remains unclear. We recently established an intestinal rehabilitation unit (IRU) and aimed to provide our experience on the current management and outcomes of IF in Iran.

Methods: In this cross-sectional case series, data were collected from an established database on IF in the Shiraz Transplant Center in Abu Ali Sina hospital, affiliated to Shiraz University of Medical Sciences, Iran from January 2018 to October 2018.

Results: Overall, 30 patients with a mean age of $44.13 \pm 10.32$ years, which included 25 males, were recruited. Short bowel syndrome (SBS) $(60 \%)$ and enterocutaneous fistulae $(27 \%)$, as complication of previous surgeries, were the main causes of IF. The most common type of IF was type $3(67 \%)$. Mesenteric ischemia was the leading mechanism of IF $(47 \%)$. Fifteen patients (50\%) received autologous gastrointestinal reconstruction surgery (AGIRS), and two (7\%) patients had serial transverse enteroplasty (STEP). At the end of follow-up, 15 patients recovered from IF (50\%). The overall survival rate was $83.3 \%$.

Conclusion: This series introduced the results of a multidisciplinary program for the treatment of IF in a middle-income country that lacks facilities for HPN. Our protocol of care, understanding the need for development of HPN, showed promising clinical outcomes.

Keywords: Home Parenteral Nutrition, Intestinal Failure, Intestinal Transplant, Iran, Middle East

Cite this article as: Nikoupour H, Arasteh P, Shamsaeefar A, Shafiekhani M, Moradi AM, Karami MY, et al. Surgical management of intestinal failure; initial experiences from an intestinal failure, rehabilitation and transplant unit in iran. Arch Iran Med. 2021;24(4):289-295. doi: 10.34172/aim.2021.40
\end{abstract}

Received: October 1, 2019, Accepted: September 20, 2020, ePublished: April 1, 2021

\section{Introduction}

Intestinal failure (IF) is a complex condition, which is defined as reduction in gut flow in which absorption of nutrients is disrupted to a degree that necessitates intravenous supplementation in order to maintain health and growth. ${ }^{1}$ Difficulties in accessing health services, severity of the disease at presentation and the absence of adequate guidelines for early, mid-term and long-term management of this condition add to the complexities when facing patients with IF.,

Middle-income countries (MICs) are a very diverse group with regard to age distribution, population size and income, and include small nations to some of the largest countries in the world. Iran is considered to be an uppermiddle income country according to the definition by the World Bank classification ${ }^{4}$. Low- and middle-income countries mainly lack facilities for home parenteral nutrition (HPN), considered the gold standard treatment for IF, which complicates treatment of the condition in these countries. ${ }^{5}$
Intestinal rehabilitation refers to a multidisciplinary process aimed at improving intestinal function so that patients will not require parenteral nutrition $(\mathrm{PN})$ and will minimize future need for intestinal transplantation. ${ }^{6}$ This comprehensive approach in IF uses nutrition, pharmacology, and in some cases, non-transplant surgical reconstructions to achieve this objective.

We have recently established an intestinal rehabilitation unit (IRU) in Abu Ali Sina hospital, affiliated to Shiraz University of Medical Sciences, Iran. In this center, we provide specific IF rehabilitation and transplant programs which aim to provide state-of-the-art care for adult patients with different degrees of intestinal insufficiency and IF. Here, we report our first experience on the management of IF in our IRU in Iran.

\section{Materials and Methods Study Settings and Patient Selection}

In this cross-sectional case series study, data were collected from an established database on IF in the Shiraz 
Transplant Center in Abu Ali Sina hospital, affiliated to Shiraz University of Medical Sciences, Iran. All patients diagnosed with IF from January 2018 to October 2018 were included.

Data on patients' characteristics, diagnosis and type of IF, cause of IF, number of surgeries received before referral, type of operation performed for intestinal rehabilitation, and postsurgical complications based on the ClavienDindo classification of surgical complications ${ }^{7}$ were collected for each patient. The total number of patients who were weaned off PN, who were discharged and the number of patients achieving intestinal adaptation and survival at the end of follow-up were also recorded.

\section{Pre-operative Care}

After clinical and metabolic conditions were stabilized in patients, preoperative hospital PN management in patients with type II IF included the following steps:

1. Avoiding hydro-electrolytic imbalance and dehydration by use of anti-secretory and anti-motility drugs to decrease ostomy losses,

2. Improving nutritional conditions,

3. Completing an antifungal and antibiotic therapy course when needed,

4. Improving the abdominal wall condition,

5. Physical, motor and psychosocial rehabilitation,

6. Planning for autologous gastrointestinal reconstruction surgery (AGIRS).

\section{Autologous Gastrontestinal Reconstruction Surgery (AGIRS)}

AGIRS was always performed three to six months after the last surgery a patient had. If patients were not candidates for surgical or medical rehabilitation and/or developed serious complications related to the nutritional support, they would become candidates for intestinal transplantation.

AGIRS was done with the aim of resecting intestinal fistulae complexes, closing ostomies, and restoring intestinal continuity in order to preserve or increase the intestinal length. When possible, the ileocecal valve was preserved to favor intestinal adaptation. Sometimes, more than one procedure was needed to achieve a complete gastrointestinal reconstruction. AGIRS has several benefits including restoration of intestinal continuity, reducing dysfunctional bowel segments, reducing bacterial overgrowth, and improving motility. 8,9

Serial transverse enteroplasty (STEP), a procedure described by Kim et al in 2003, requires having a dilated intestinal segment of more than $5 \mathrm{~cm}$ in order to performed subtotal transection of the intestine perpendicular to the main longitudinal axis, using multiples linear laparoscopic triple line staplers alternately placed at $2 \mathrm{~cm}$ intervals, depending on the length of the intestine requiring enteroplasty. ${ }^{9,10}$

\section{Post-operative Care}

All patients were admitted to the intensive care unit for immediate post-operative care. Once hemodynamic instability was treated, $\mathrm{PN}$ was started using pre-operative formulation. On the 5th post-operative day, a small bowel follow-through with water-soluble contrast and methylene blue or an abdominal CT scan with intravenous and oral contrast was performed. This was done to verify the indemnity of the intestinal anastomosis in order to initiate oral or gastrointestinal intake, as well as to rule out the presence of any residual intra-abdominal fluid collection. If oral intake of clear fluids was tolerated for 24 hours, diet was progressed to a low residue diet. Patients received anti-secretory (ranitidine/omeprazole) and anti-motility agents (loperamide) or cholestyramine when indicated. Once the oral and/or enteral intake reached $60 \%$ of the patient's nutritional requirements, the volume and caloric intake of PN formulation were decreased. When the oral/ enteral intake covered $75 \%$ of the requirements, $\mathrm{PN}$ was suspended. It was considered that the patient recovered intestinal sufficiency when he was capable of maintaining weight and hydration for six months without any form of PN support.

\section{Follow-up}

For the first year after surgery, patients were visited every month. After the first year of surgery, patients were given annual follow-ups. In cases when patients did not refer for their follow-up, they were contacted and in cases of no answer, their first of kin was contacted and information regarding the patient's condition (death, hospitalization etc) was acquired and another visit was appointed. In case of development of complications, patients were advised to refer to the transplantation center even without prior appointed visitations.

\section{Statistical Analysis}

The statistical analyses were carried out using the Statistical Package for Social Sciences software (IBM SPSS Inc. version 20, Chicago, IL, USA ${ }^{\oplus}$ ). Quantitative data with normal distribution are presented as means and standard deviation (SD) and those without normal distribution are presented as median and interquartile range (IQR). Qualitative data are presented as frequency and percentage. All values have been rounded. For means, values have been rounded to one decimal place and for percentages, values have been rounded to include no decimals.

\section{Results}

Thirty patients with a mean age of $44.13 \pm 10.32$ years were included in the study. Five patients were women (17\%) and 25 (83\%) were men.

Short bowel syndrome (SBS) was the main diagnosis for IF $(60 \%)$ followed by enterocutaneous fistulae $(27 \%)$. One patient $(3 \%)$ presented with chronic intestinal 
pseudo-obstruction; furthermore, one patient (3\%) was admitted with prolonged ileus and two patients (7\%) were hospitalized with IF post distal pancreatectomy with normal intestinal length.

Regarding the primary etiology of IF, the most common mechanism was mesenteric ischemia (47\%), followed by post-surgical (more commonly after colon cancer) (33\%).

The most common types of IF were type III and type II, which were seen in $67 \%$ and $27 \%$ of patients, respectively.

Regarding the conditions for transplantation, the majority of patients were not candidates for any type of transplantation (70\%). Moreover, 4 patients (13\%) were candidates for isolated intestinal transplantation, two patients (7\%) for multi-visceral transplantation, and two patients (7\%) were candidates for isolated pancreas transplantation.

The median (IQR) length of hospital stay was 59 (35, 84 ) days (range 20-240 days). The median (IQR) number of operations done for patients was $3(1.75,4)$.

All the included patients received $\mathrm{PN}$ as standard therapy. Fifteen patients (50\%) had AGIRS (Figure 1). The STEP procedure was performed in two patients (7\%). Regarding complications, according to the Clavien-Dindo classification index, 8 patients $(27 \%)$ had type 1,13 patients (43\%) had type 2, 2 patients (7\%) had type 3, 2 patients $(7 \%)$ had type 4 , and 5 patients (17\%) developed type 5 complications. The most common in-hospital complications included catheter infections (47\%), sepsis (20\%), and renal failure (10\%).

At the end of the follow-up, 16 patients (53\%) had recovered from IF and were discharged from the hospital (Figure 2). Moreover, four patients (13\%) were candidates for re-reconstruction, five patients (17\%) were candidates for transplantation, including two (7\%) for pancreas transplantations and three $(10 \%)$ for intestinal transplantations. Overall, five (17\%) patients died during the study period. Table 1 shows the patients' baseline and

Table 1. Characteristic of Patients with Intestinal Failure*

\begin{tabular}{lc}
\hline Variables & Statistics \\
\hline Age, Mean \pm SD & $44.1 \pm 10.3$ \\
\hline Sex, No. (\%) & $25(83)$ \\
Male & $5(17)$ \\
Female & \\
\hline Secondary etiology of IF, No. (\%) & $18(60)$ \\
Short bowel syndrome & $8(27)$ \\
Enterocutaneous fistula & $1(3)$ \\
Chronic intestinal pseudo-obstruction & $1(3)$ \\
Prolonged ileus & $2(7)$ \\
Unknown & $14(47)$ \\
\hline Primary etiology of IF, No. (\%) & $10(33)$ \\
\hline Mesenteric ischemia & $2(7)$ \\
Post-surgery & $3(10)$ \\
\hline Liver transplantation/portal vein thrombosis & \\
Colon cancer & \\
\hline
\end{tabular}

Table 1. Continues

\begin{tabular}{|c|c|}
\hline Variables & Statistics \\
\hline Gastric cancer & $1(3)$ \\
\hline Sleeve gastrectomy & $1(3)$ \\
\hline Fistula/appendectomy & $1(3)$ \\
\hline Total pancreatectomy & $2(7)$ \\
\hline Volvulus & $1(3)$ \\
\hline Chronic intestinal pseudo-obstruction & $1(3)$ \\
\hline Trauma & $1(3)$ \\
\hline Multiple resections & $1(3)$ \\
\hline Familial adenomatous polyposis & $1(3.3)$ \\
\hline Perforated duodenal ulcer & $1(3)$ \\
\hline \multicolumn{2}{|l|}{ Type of intestinal failure, No. (\%) } \\
\hline 1 & $2(7)$ \\
\hline 2 & $8(27)$ \\
\hline 3 & $20(67)$ \\
\hline \multicolumn{2}{|l|}{ Hospital stay, days } \\
\hline Mean \pm SD & $69.0 \pm 48.3$ \\
\hline Median (IQR) & $59(35,84)$ \\
\hline Number of operations, Median (IQR) & $3.00(1.75,4)$ \\
\hline \multicolumn{2}{|l|}{ Catheter infection, No. (\%) } \\
\hline Yes & $14(47)$ \\
\hline No & $16(53)$ \\
\hline \multicolumn{2}{|l|}{ Sepsis, No. (\%) } \\
\hline Yes & $6(20)$ \\
\hline No & $24(80)$ \\
\hline \multicolumn{2}{|l|}{ Liver failure, No. (\%) } \\
\hline Yes & $1(3)$ \\
\hline No & $29(97)$ \\
\hline \multicolumn{2}{|l|}{ Diabetes mellitus, No. (\%) } \\
\hline Yes & $2(7)$ \\
\hline No & $28(93)$ \\
\hline \multicolumn{2}{|l|}{ Pneumonia, No. (\%) } \\
\hline Yes & $1(3)$ \\
\hline No & $29(97)$ \\
\hline \multicolumn{2}{|l|}{ Renal failure, No. (\%) } \\
\hline Yes & $3(10)$ \\
\hline No & $27(90)$ \\
\hline \multicolumn{2}{|l|}{ Type of operation, No. (\%) } \\
\hline Autologous gastrointestinal Reconstruction & $15(50)$ \\
\hline Serial transverse enteroplasty & $2(7)$ \\
\hline Resection and anastomosis & $13(43)$ \\
\hline \multicolumn{2}{|l|}{ Follow-up outcomes, No. (\%) } \\
\hline Discharged and recovered IF & $16(53)$ \\
\hline Candidate for re-reconstruction & $4(13)$ \\
\hline Transplantation candidate & $5(17)$ \\
\hline Bowel transplantation & $3(10)$ \\
\hline Pancreas transplantation & $2(7)$ \\
\hline Died & $5(17)$ \\
\hline \multicolumn{2}{|l|}{ Mortality, No. (\%) } \\
\hline Yes & $5(17)$ \\
\hline No & $25(83)$ \\
\hline
\end{tabular}

*All values have been rounded. For means, values have been rounded to one decimal place and for percentages values have been rounded to include no decimals 

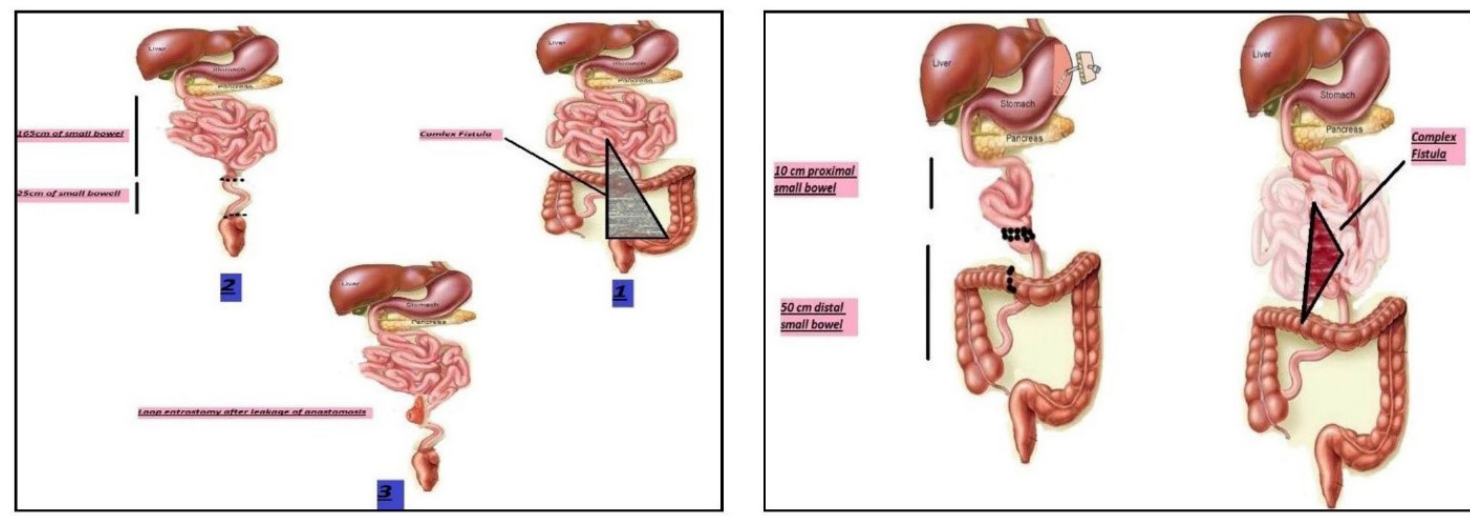

Figure 1. Schematic View of Complex Fistula Closure Using AGIRS Procedure in Two Patients.
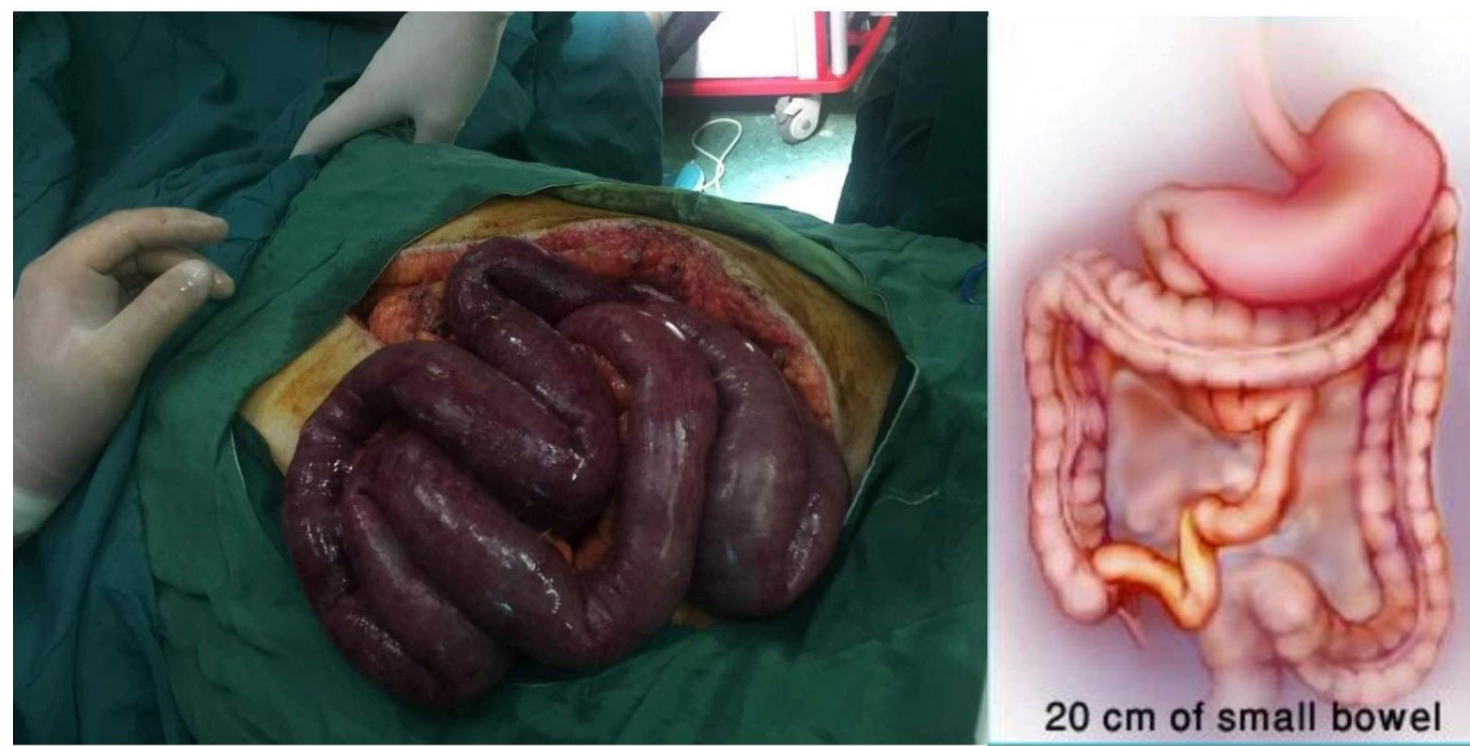

Figure 2. A 37-year-old man who had developed portal vein thrombosis post-splenectomy and nearly total small bowel resection, referred to our center. The patient was hospitalized in the IRU and was on PN for 2 months. Work-up and surgical exploration showed $5 \mathrm{~cm}$ of proximal jejunum and $15 \mathrm{~cm}$ of distal ileum including ICV and full length of the colon. Primary anastomosis was done following which the patient was on PN for 1-month post-surgery. The patient was then weaned off $\mathrm{PN}$ and discharged in good conditions and with regular follow-up.

clinical characteristics in our study.

\section{Discussion}

In this study, we described our experience with 30 patients with IF. To the best of the authors' knowledge, this is the first report from the Middle East on the outcomes and management of patients with IF in an IRU without facilities for HPN. Data published regarding the development of intestinal rehabilitation programs are scarce in Asia.

The first form of PN was established in Shiraz and was entitled the "hyperalimentation unit" in Sadi hospital affiliated to Shiraz University of Medical Sciences in $1972 .{ }^{11}$ Considering the high costs of intestinal transplantations and the non-existence of facilities for HPN, we established the first IRU in our region.

According to the European Society for Parenteral and Enteral Nutrition (ESPEN), the acute form of IF is categorized into two forms of functional and pathophysiological.

IF is "pathophysiologically" classified into five groups: short bowel (SB) with reduced absorptive mucosal surface; intestinal fistula caused by massive bypass of absorptive mucosal surface; intestinal dysmotility that is restricted oral/enteral nutrition or total fasting from intolerance due to feeding-related exacerbation of digestive symptoms or due to episodes of non-mechanical intestinal obstruction; mechanical obstruction; extensive SB mucosal disease, which is referred to as inefficient absorption and/or nutrient loss of mucosal surface. ${ }^{12,13}$

Patients with type 1 and most patients with type 2 IF recover fully over time. Type 3 IF is mainly observed in SBS cases which requires long-term PN. ${ }^{14}$

In the case of SBs, EC fistula, or extensive SB disease, malabsorption can be considered the primary mechanism. In the case of intestinal dysmotility or intestinal mechanical obstruction, IF can be mainly attributed to oral/enteral nutrition blockade or total oral fasting.

SBS is the main reason for type $3 \mathrm{CIF}$, which involves about $75 \%$ of adults and $50 \%$ of children on HPN in Europe. ${ }^{15}$ There is a high risk for development of IF in 
adults with $\mathrm{SB}<35 \mathrm{~cm}$ with a jejunoileal anastomosis with an intact colon, $<60 \mathrm{~cm}$ with a jejunocolic anastomosis or $<115 \mathrm{~cm}$ with an end-jejunostomy. ${ }^{16} \mathrm{HPN}$ can be weaned off in $50 \%$ of adult patients following medical and surgical treatments. Complete HPN wean-off is almost unlikely $(<10 \%)$ in patients with SBS and almost always relapses after 2-3 years due to intestinal abduction. ${ }^{17-19}$

Enteric fistulas are classified as internal (leak to the gastrointestinal tract or other organs such as the bladder and vagina) and external (leak to skin). The physiologic classification of enterocutaneous fistulas are as follows: low-output fistulas ( $<200 \mathrm{~mL} / \mathrm{d}$ drainage); moderateoutput fistulas (200-500 mL/d drainage); high-output fistulas $(>500 \mathrm{~mL} / \mathrm{d}$ drainage $)$. Although various etiologies can lead to enteric fistula formation, the most common etiologies include distal obstruction, radiation, foreign body, inflammation or infection, neoplasia, and epithelialization. Approximately one third of all EC fistula cases are comorbidities associated with Crohn's disease. ${ }^{20,21}$ One of the main causes of type 2 IF in adults is EC fistulas (Figure 3). PN, which may cause metabolic instability, plays a vital role in the initiation of treatment in these patients. ${ }^{12}$ Spontaneous closure rates vary depending on the cause and volume of the fistula. ${ }^{22,23}$ About 33\% of EC fistula cases improve spontaneously within five to six weeks with conservative measures. ${ }^{24-26}$ Generally, lowoutput, long-track, and proximal SB are most likely to heal with conservative measures. Patients with enterocutaneous fistulae who have failed 5 to 6 weeks of non-operative management will likely need surgery. ${ }^{27}$

The incidence of IF varies amongst European and South American countries. Prasad and Pollard ${ }^{28}$ reported the annual incidence rate of the condition to be 2.6 patients for the UK, 13.9 for Denmark, and 80-200 for the United States per one million individuals. Moreover, the incidence rate was reported to be 1.1-12.7 per million adults in Europe. According to a recent report from the Dutch registry for Intestinal Failure and Intestinal Transplantation in 2013, the prevalence of IF showed a remarkable rise during the past decades. ${ }^{28-31}$

All the 42 Latin American and Caribbean countries have patients diagnosed with IF, but few (6 out of 42, 16.66\%) are currently capable of providing sustained and complete care for IF including HPN and intestinal transplantation. ${ }^{5}$ Postsurgical complications followed by mesenteric ischemia are the main causes of IF for adults in Latin America and the Caribbean region. The main etiology for IF in high-income countries are Crohn's disease followed by mesenteric ischemia, and postsurgical complications. ${ }^{5}$

The most common indication for PN in Asian adults is SBS secondary to mesenteric ischemia, postsurgical complications, followed by cancer. Inflammatory bowel diseases are an unusual cause of PN in Asia compared to the United States and Europe. ${ }^{32}$

With regard to our results, the most common indication

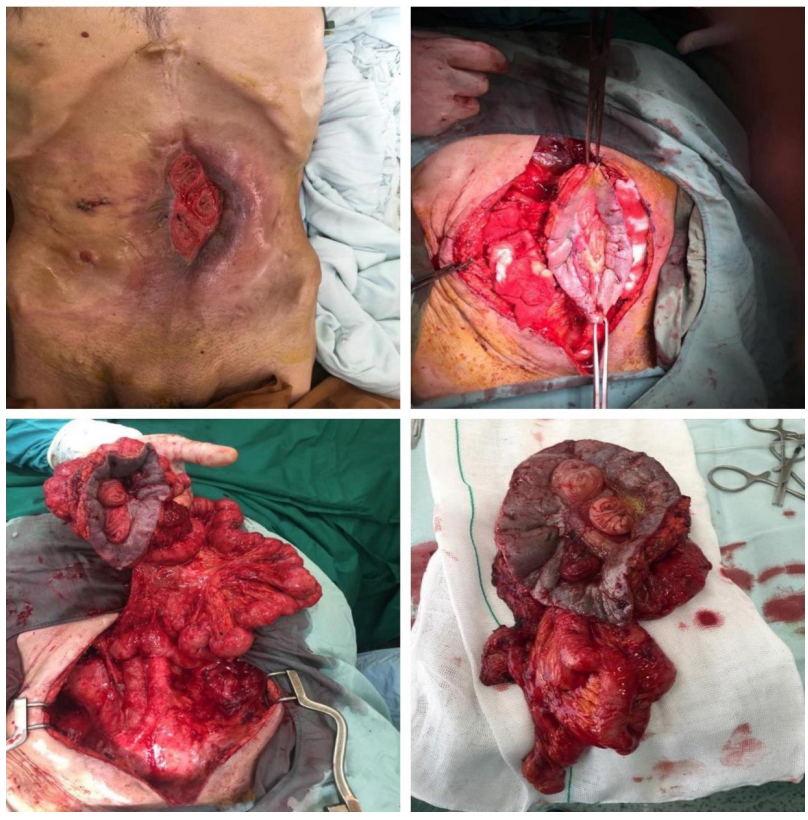

Figure 3. A 60-year-old man who had undergone colon surgery for colon cancer and had multiple operations due to fistula formation, referred to us with a picture of island of fistulas. The patients underwent exploration en-bloc resection of the fistula complex with remnants of total small bowel length of $150 \mathrm{~cm}$; moreover, end-ileostomy was performed. The patient came off $\mathrm{PN}$ and is in good conditions.

for PN in Iranian adults was SBS secondary to mesenteric ischemia followed by post-surgical complications.

AGIRS and STEP were the most common surgical procedures performed among adults and children with IF, respectively (Figure 4). The first multidisciplinary IF rehabilitation and transplantation program was established in Latin America in 2006 in Argentina and Colombia. One report from Buenos Aires, Argentina showed that a total of $74.1 \%$ of patients with intestinal remnants of more than $50 \mathrm{~cm}$ after AGIRS were able to accomplish intestinal autonomy within 5 years after the operation. ${ }^{5}$

Although not precise, as no national registration program exists, the rate of SB transplantation is somewhat higher in Iran compared to other Asian countries. ${ }^{5}$ This can be due to two differences between Iran and other Asian countries: first is that unlike Iran, most South Eastern Asian countries are capable of providing HPN for their patients, and the second is due to the popularity of organ donation in Iran and better access to an organ pool of deceased donors as a result of social and cultural factors, which has caused patients in Iran to shift towards SB transplantation.

It is difficult to find reports on the existence of $\mathrm{HPN}$ in countries in Asia and few reports of successful HPN exist in Taiwan, Japan and China. ${ }^{5,33}$

This study was not without limitations. As we do not have a national registration system, we do not have information regarding the exact number of patients with IF in our country. We have established a multidisciplinary team that applies our treatment protocol which includes intestinal transplantation if needed; other centers with 

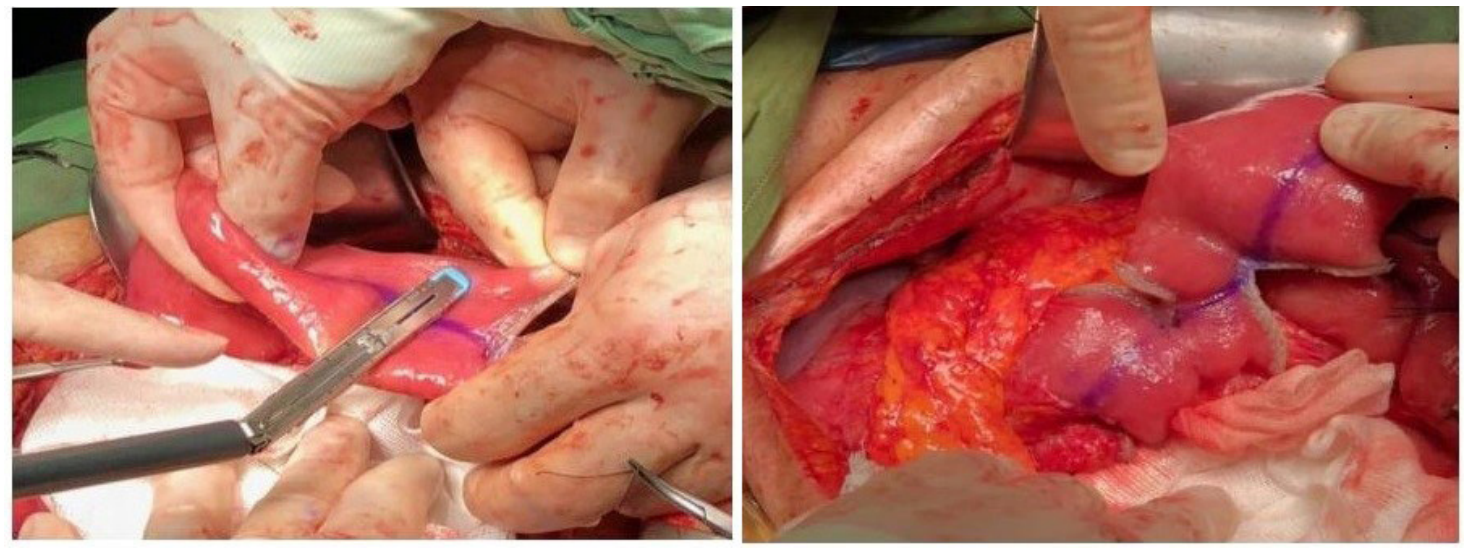

Figure 4. A 55-year-old man who had been sent to the operation room with diagnosis of bowel gangrene post-laminectomy in one of our nearby hospitals. Resection of gangrenous sections was performed; the remnant was proximal $30 \mathrm{~cm}$ of small bowel, which was stapled and put into the abdominal cavity, colon just distal to mid transverse, with a gastroduodenostomy tube for proximal decompression. The patient was then hospitalized in the IRU and was on TPN for 2 months, after which reconstructive surgery was planned. During surgery, both the length and diameter of the small bowel had increased (length $55 \mathrm{~cm}$ and diameter $5 \mathrm{~cm}$ ). So, the STEP procedure was done and the length was increased to $90 \mathrm{~cm}$. After 1 month of postoperative PN, the patient came off PN and was discharged with regular follow-up.

limited resources may not be able to completely apply our protocol, although most of our patients were spared from transplantation using reconstructive surgeries. Considering that we have just recently established our IRU, the follow-up of our patients was limited by nature and longer follow-ups will better show the long-term complications and outcomes.

In conclusion, by establishing the first IRU in our center and practicing AGIR, we have been able to achieve intestinal autonomy in patients suffering from IF, reduce the number of candidates for intestinal transplantation, and reduce mortality rates. Our report shows a successful experience with management of patients with IF by establishing an IF, rehabilitation and transplant unit in a country that lacks facilities for HPN.

\section{Clinical Relevancy Statement}

We have recently established an IRU in Abu Ali Sina Hospital affiliated to Shiraz University of Medical Sciences, Iran. In this center, we provide specific IF, rehabilitation and transplant programs which aim to provide state-of-the-art care for adult patients with different degrees of intestinal insufficiency and IF. To the best of our knowledge, this is the first report on IF patients, their management and outcomes in Iran, a country that lacks facilities for HPN which is considered to be the gold standard treatment of this disease.

\section{Authors' Contribution}

HN, AS, MS, GEG, and SN aided in study concpetualization. HN, HE, AMM, MYK, and SN aided in study design and data gathering. PA Aided in statistical analysis. HN, MS, AS and PA aided in interpretation of results. HN, MYK, PA and MS aided in prepration of manuscript draft. All authors aided in critical revision of final manuscript and approved the final format.

\section{Conflict of Interest Disclosures}

There is no conflict of interest to be declared regarding the manuscript.

\section{Ethical Statement}

The study protocl was approved by the Institutional Review Board of Shiraz University of Medical Sciences, Shiraz, Iran. All study protocols followed guidelines stated in the Declaration of Helsinki and the declaration of Istanbul.

\section{References}

1. Fraser C. Intestinal failure and short bowel syndrome. Medicine. 2019;47(5):320-4. doi: 10.1016/j.mpmed.2019.02.006.

2. Pironi L, Konrad D, Brandt C, Joly F, Wanten G, Agostini F, et al. Clinical classification of adult patients with chronic intestinal failure due to benign disease: An international multicenter cross-sectional survey. Clin Nutr. 2018;37(2):72838. doi: 10.1016/j.clnu.2017.04.013

3. Fishbein TM, Gondolesi GE, Kaufman SS. Intestinal transplantation for gut failure 1. Gastroenterology. 2003;124(6):1615-28. doi :10.1016/S0016-5085(03)00375-5

4. Bank TW. World Bank Country and Lending Groups 2020. Available from: https://datahelpdesk.worldbank.org/ knowledgebase/articles/906519-world-bank-country-andlending-groups.

5. Gondolesi GE, Pattín F, Nikkoupur H. Management of intestinal failure in middle-income countries, for children and adults. Curr Opin Organ Transplant. 2018;23(2):212-8. doi: 10.1097/MOT.0000000000000512.

6. DiBaise JK, Young RJ, Vanderhoof JA. Intestinal rehabilitation and the short bowel syndrome: part 1. Am J Gastroenterol. 2004;99(7):1386-95. doi: 10.1111/j.15720241.2004.30345.x.

7. Dindo D, Demartines N, Clavien PA. Classification of surgical complications: a new proposal with evaluation in a cohort of 6336 patients and results of a survey. Ann Surg. 2004;240(2):20513. doi: 10.1097/01.sla.0000133083.54934.ae.

8. Sudan D, Rege A. Update on surgical therapies for intestinal failure. Curr Opin Organ Transplant. 2014;19(3):267-75. doi: 10.1097/MOT.0000000000000076.

9. Thompson J, Sudan D. Intestinal lengthening for short bowel syndrome. Adv Surg. 2008;42:49-61. doi: 10.1016/j. yasu.2008.03.009.

10. Kim HB, Fauza D, Garza J, Oh JT, Nurko S, Jaksic T. Serial transverse enteroplasty (STEP): a novel bowel lengthening procedure. J Pediatr Surg. 2003;38(3):425-9. doi: 10.1053/ jpsu.2003.50073.

11. Parsa MH, Habif DV, Ferrer JM, Lipton R, Yoshimura NN. 
Intraveneous hyperalimentation: indications, technique, and complications. Bull N Y Acad Med. 1972;48(7):920-42.

12. Pironi L, Arends J, Baxter J, Bozzetti F, Peláez RB, Cuerda $C$, et al. ESPEN endorsed recommendations. Definition and classification of intestinal failure in adults. Clin Nutr. 2015;34(2):171-80. doi: 10.1016/j.clnu.2014.08.017.

13. Khan S, Gatt M, Horgan A, Anderson I, MacFie J. Guidelines for implementation of enhanced recovery protocols. Issues in Professional Practice. 2009

14. Grainger JT, Maeda Y, Donnelly SC, Vaizey CJ. Assessment and management of patients with intestinal failure: a multidisciplinary approach. Clin Exp Gastroenterol. 2018;11:233-41. doi: 10.2147/CEG.S122868.

15. Pironi L, Hébuterne $X$, Van Gossum A, Messing B, Lyszkowska $\mathrm{M}$, Colomb V, et al. Candidates for intestinal transplantation: a multicenter survey in Europe. Am J Gastroenterol. 2006;101(7):1633-43; quiz 1679. doi: 10.1111/j.15720241.2006.00710.x

16. Nightingale J, Woodward JM. Guidelines for management of patients with a short bowel. Gut. 2006;55(suppl 4):iv1-iv12. doi: 10.1136/gut.2006.091108.

17. Pironi L, Goulet O, Buchman A, Messing B, Gabe S, Candusso $M$, et al. Outcome on home parenteral nutrition for benign intestinal failure: a review of the literature and benchmarking with the European prospective survey of ESPEN. Clin Nutr. 2012;31(6):831-45. doi: 10.1016/j.clnu.2012.05.004.

18. Messing B, Crenn P, Beau P, Boutron-Ruault MC, Rambaud JC, Matuchansky $\mathrm{C}$. Long-term survival and parenteral nutrition dependence in adult patients with the short bowel syndrome. Gastroenterology. 1999;117(5):1043-50. doi: 10.1016/S00165085(99)70388-4.

19. Pironi L, Joly F, Forbes A, Colomb V, Lyszkowska M, Baxter J, et al. Long-term follow-up of patients on home parenteral nutrition in Europe: implications for intestinal transplantation. Gut. 2011;60(1):17-25. doi: 10.1136/gut.2010.223255.

20. Schecter WP, Hirshberg A, Chang DS, Harris HW, Napolitano LM, Wexner SD, et al. Enteric fistulas: principles of management. J Am Coll Surg. 2009;209(4):484-91. doi: 10.1016/j.jamcollsurg.2009.05.025.

21. Hollington P, Mawdsley J, Lim W, Gabe S, Forbes A, Windsor A. An 11-year experience of enterocutaneous fistula. Br J Surg. 2004;91(12):1646-51. doi: 10.1002/bjs.4788.

22. Campos AC, Andrade DF, Campos GM, Matias JE, Coelho
JC. A multivariate model to determine prognostic factors in gastrointestinal fistulas1. J Am Coll Surg. 1999;188(5):483-90. doi: 10.1016/S1072-7515(99)00038-1.

23. Fazio VW, Coutsoftides T, Steiger E. Factors influencing the outcome of treatment of small bowel cutaneous fistula. World J Surg. 1983;7(4):481-8. doi: 10.1007/BF01655937.

24. Aguirre A, Fischer JE, Welch CE. The role of surgery and hyperalimentation in therapy of gastrointestinalcutaneous fistulae. Ann Surg. 1974;180(4):393-401. doi: 10.1097/00000658-197410000-00003.

25. Prickett D, Montgomery R, Cheadle WG. External fistulas arising from the digestive tract. South Med J. 1991;84(6):7369. doi: 10.1097/00007611-199106000-00015.

26. Schein M. What's new in postoperative enterocutaneous fistulas?. World J Surg. 2008;32(3):336-8. doi: 10.1007/ s00268-007-9411-x.

27. Visschers RG, van Gemert WG, Winkens B, Soeters PB, Damink SWO. Guided treatment improves outcome of patients with enterocutaneous fistulas. World J Surg. 2012;36(10):2341-8. doi: 10.1007/s00268-012-1663-4.

28. Prasad KR, Pollard S. Small bowel transplantation. Curr Opin Gastroenterol. 2000;16(2):126-33. doi: 10.1097/00001574200003000-00006.

29. Roskott AM, Groen H, Rings EH, Haveman JW, Ploeg RJ, Serlie MJ, et al. Cost-effectiveness of intestinal transplantation for adult patients with intestinal failure: a simulation study. Am J Clin Nutr. 2014;101(1):79-86. doi: 10.3945/ajcn.114.083303.

30. Neelis E, Roskott A, Dijkstra G, Wanten G, Serlie M, Tabbers $M$, et al. Presentation of a nationwide multicenter registry of intestinal failure and intestinal transplantation. Clin Nutr. 2016;35(1):225-9. doi: 10.1016/j.clnu.2015.01.010.

31. Health Quality O. Small bowel transplant: an evidence-based analysis. Ont Health Technol Assess Ser. 2003;3(1):1-72.

32. Wang MY, Wu MH, Hsieh DY, Lin J, Lee PH, Chen WJ, et al. Home parenteral nutrition support in adults: experience of a medical center in Asia. JPEN J Parenter Enteral Nutr. 2007;31(4):306-10. doi: 10.1177/0148607107031004306.

33. Wu G, Jiang $Y$, Zhu $X$, Jin D, Han $Y$, Han J, et al. Prevalence and risk factors for complications in adult patients with short bowel syndrome receiving long-term home parenteral nutrition. Asia Pac J Clin Nutr. 2017;26(4):591-7. doi: 10.6133/ apjen.082016.08. 